\title{
Evaluation of the Dehydration Performance of Zeolite NaA Membrane on Porous Alumina Tube by the Alumina X-ray Diffraction Intensity
}

\author{
Tomohiro Kyotani, ${ }^{\dagger}$ Soushi Inoue, Sonoko KaKui, and Junji Saito \\ Bussan Nanotech Research Institute, Inc., Mitsui \& Co. Ltd. Nanotech Park, \\ 2-1, Koyadai, Tsukuba, Ibaraki 305-0074, Japan
}

\begin{abstract}
A zeolite NaA (A-type zeolite of $c a .0 .4 \mathrm{~nm}$ pore size; Linde Type A, LTA) membrane for the dehydration of alcohol was characterized by X-ray diffraction analysis (XRD). Also, the relationship between the X-ray absorption and the EtOH/ $\mathrm{H}_{2} \mathrm{O}$ pervaporation (PV) dehydration performance (water selectivity and permeation flux) of the LTA membrane was first investigated. The LTA membranes used here were gel-synthesized hydrothermally on an alumina porous support tube. Since diffraction lines from the alumina generate from a deeper layer than those of the LTA crystal, and are absorbed by both the surface LTA crystal and materials embedded in the alumina porous support, the alumina (113) diffraction line was intensively monitored to estimate the overall X-ray absorption by the LTA membrane. The intensity of the alumina (113) diffraction line showed a good correlation with the PV dehydration performance of the LTA membrane, that is, lower values with the water selectivity and higher values with the permeation flux. The lower diffraction intensity means stronger X-ray absorption by the LTA membrane. The major factor causing the difference in the X-ray absorption is the thickness or quantity of materials embedded in an alumina porous support, rather than those of the surface LTA crystal. These phenomena can be used conveniently (without real PV experiments) to determine the EtOH/ $/ \mathrm{H}_{2} \mathrm{O}$ PV dehydration performance of the LTA membrane.
\end{abstract}

(Received October 5, 2005; Accepted December 12, 2005)

\section{Introduction}

A zeolite $\mathrm{NaA}$ (A-type zeolite of $c a .0 .4 \mathrm{~nm}$ pore size; Linde Type A, LTA) membrane supported by an alumina porous support tube is a useful tool for the dehydration of organic liquids. ${ }^{1}$ For designing not only the synthesis method of the LTA membrane, but also the optimum dehydration process, it is very important to understand a certain relationship between the LTA membrane structure and the dehydration performance. However, since many characterizations of the LTA membrane have been carried out using mainly chemical engineering methods, such a relationship has been little clarified. ${ }^{1,2}$ In the case of developing a practical nondestructive analytical method, it is better to use bulk analysis rather than microscopic analysis, like SEM and TEM, because it is very difficult to judge the overall information from micro and nano area analysis. Therefore, as a part of an analytical study of the LTA membrane, we have already reported a method using Fourier transform infrared attenuated total reflectance (FTIR-ATR), and discussed the relationship between the $\mathrm{Si}-\mathrm{O}$ spectral shape of the membrane and the $\mathrm{EtOH} / \mathrm{H}_{2} \mathrm{O}$ pervaporation (PV) dehydration performance (water selectivity and permeation flux) as well as its application to a nondestructive and rapid evaluation of the PV performance. ${ }^{3}$ In our previous work, ${ }^{3,4}$ it was suggested that not only the surface LTA crystal, but also materials embedded in the alumina porous support affect the PV performance. However, since the FTIR-ATR method detects only the relatively upper layer of the membrane, information on

\footnotetext{
$\doteqdot$ To whom correspondence should be addressed.

E-mail: t-kyotani@xnri.com
}

materials embedded in deep parts of the alumina porous support may not be very much. In the present report, especially, to discuss such materials and to develop a useful method by which the PV performance can be determined without real PV experiments, the LTA membrane was characterized by X-ray diffraction analysis (XRD) and the relationship between X-ray absorption and water permeation behavior was considered for the first time. Consequently, a simple and useful method using the XRD was proposed to conveniently estimate the EtOH/ $\mathrm{H}_{2} \mathrm{O}$ PV dehydration performance of the LTA membrane.

\section{Experimental}

Tubular LTA membrane sample and PV test of the EtOH/H $\mathrm{H}_{2} \mathrm{O}$ mixture

Figure 1 shows a schematic diagram of a tubular LTA membrane and a TEM image of a cross-section thin-layer prepared by a focused ion beam (FIB). ${ }^{4}$ This time, the cross section thin-layer specimen by FIB was prepared up to about 15 $\mu \mathrm{m}$ depth, because the alumina support layer is very hard, and thus FIB operation is very time-consuming. As shown in Fig. 1 (a), porous alumina tubes with dimensions of $12 \pm 0.3 \mathrm{~mm}$ o.d., $9 \pm 0.3 \mathrm{~mm}$ i.d. and $800 \mathrm{~mm}$ length were used as a substrate. ${ }^{5,6}$ The average pore size and porosity of the substrates were $1.3 \mu \mathrm{m}$ and $40-47 \%$, respectively. 5,6 LTA membranes were gel-synthesized hydrothermally on the substrates according to our previous work. ${ }^{5,6}$ The thickness of the surface layer of the LTA membranes used in this study was approximately $2-3 \mu \mathrm{m}$, as shown in Fig. 1(b).

To estimate the dehydration performance of the LTA membrane, we carried out a PV experiment according to our 


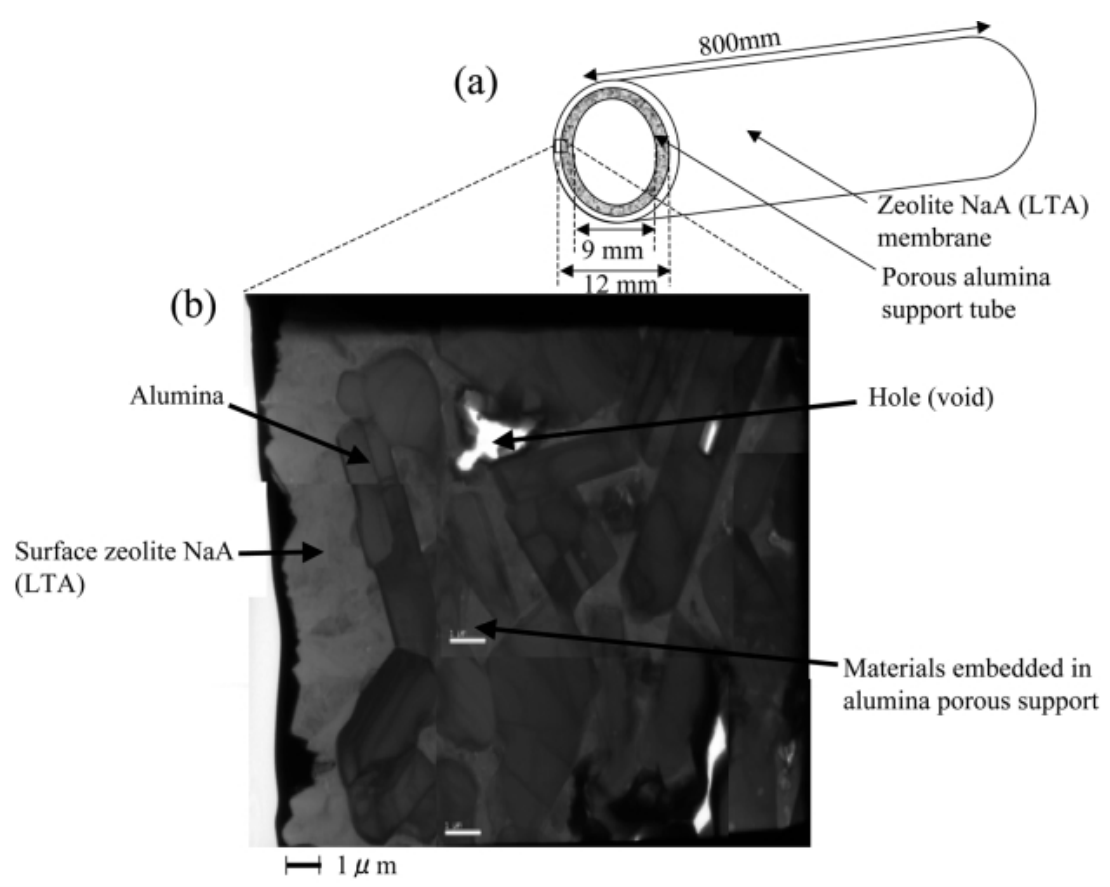

Fig. 1 Schematic diagram of tubular zeolite NaA (LTA) membrane (a) and a TEM image of the cross section (b).

previous work. ${ }^{3,5,6}$ The $800 \mathrm{~mm}$ LTA membrane tubes were cut into several $100 \mathrm{~mm}$ tubes prior to the PV test. Using the 100 $\mathrm{mm}$ tubes, feed liquid (ethanol/water: 90/10) was fed to the outer side (membrane side) of the tube, and the inside of the tube was evacuated through a vacuum line. A permeating vapor was collected by a cold trap cooled by liquid nitrogen. Composition analysis of the feed and permeate was performed on a gas chromatograph. The PV performance of the membrane tube was evaluated for the permeation flux, $Q$, in $\mathrm{kg} /\left(\mathrm{m}^{2} \mathrm{~h}\right)$, and water selectivity, $\alpha$. The selectivity of water over ethanol was defined as $\alpha_{\mathrm{w} / \mathrm{e}}=\left(Y_{\mathrm{w}} / Y_{\mathrm{e}}\right) /\left(X_{\mathrm{w}} / X_{\mathrm{e}}\right)$, where $X$ and $Y$ are the weight fractions of the water components in the binary feed mixture and in the permeate, respectively. Subscripts $w$ and e refer to water and ethanol, respectively. Therefore, the higher $\alpha$ values mean better dehydration performance.

\section{XRD measurement}

A Rigaku X-ray diffractometer (RINT 2000) with a copper tube and a nickel filter was operated at $40 \mathrm{kV}$ and $40 \mathrm{~mA}$. Normal conditions of $1^{\circ}$ and $0.3 \mathrm{~mm}$ were used for $\mathrm{D} \& \mathrm{~S}$ and $\mathrm{R}$ slits, respectively. The specimen for the XRD measurement was prepared as a $20 \mathrm{~mm}$ tube, which was cut from a $100 \mathrm{~mm}$ tube after a PV test. The $20 \mathrm{~mm}$ tubular specimen was set in a normal holder for powder samples. An X-ray beam was irradiated along the long axis of the $20 \mathrm{~mm}$ tube. X-ray diffraction patterns of the $20 \mathrm{~mm}$ tubes were measured for $2 \theta$, ranging from 10 to 50 degrees, and the alumina (113) diffraction line was intensively monitored to evaluate X-ray absorption by the LTA membrane.

\section{Results and Discussion}

As an example, Fig. 2 shows the relationships among the LTA (222) diffraction intensity and the EtOH/ $\mathrm{H}_{2} \mathrm{O}$ PV dehydration performance. As shown in Fig. 2, in this study, the XRD patterns and intensities from LTA crystal did not show a marked difference corresponding to the PV dehydration performance.
Since many previous studies also have not shown a significant relationship between the XRD patterns and the PV dehydration performance, the XRD method has not been utilized to study the water-permeation behavior in the LTA membrane. ${ }^{1,2}$ These results suggest that the surface LTA layer may not be essential for dehydration. Therefore, the diffraction lines from the alumina porous support were intensively monitored in this study and the (113) diffraction line was selected because of no overlapping with other lines and its strong relative intensity. Since the diffraction lines from the alumina are generated from deeper layer than those of the surface LTA crystal, and are absorbed by both the surface LTA crystal and materials embedded in the alumina porous support, such a diffraction line can be considered to be a good indicator to estimate the overall X-ray absorption by the LTA membrane. Figure 3 shows relationships among the alumina (113) diffraction intensity and the EtOH$/ \mathrm{H}_{2} \mathrm{O}$ PV dehydration performance. The relative standard deviations (RSDs) in the X-ray measurements of three different blank alumina tubes before hydrothermal synthesis and real LTA membrane samples were $2.2 \%$ and $2-5 \%$, respectively. Therefore, the effect of the tubular shape on the $\mathrm{X}$-ray measurement can be canceled in the case of reading the tendency of Fig. 3. As shown in Fig. 3, the intensity of the alumina (113) diffraction line showed a good correlation with the PV dehydration performance of the LTA membrane, that is, lower values with the water selectivity and higher values with the permeation flux. As an important conclusion for the purpose of this study, these relationships can be conveniently used (without a real PV test) to determine the $\mathrm{EtOH} / \mathrm{H}_{2} \mathrm{O} \mathrm{PV}$ performance of the LTA membrane.

As described above, since the blank alumina (113) intensities from original alumina supports before hydrothermal synthesis changed little among different supports, the self X-ray absorption of alumina can also be canceled in the case of reading the tendency of Fig. 3. Namely, the factor causing the alumina (113) intensity variation observed in Fig. 3 is absorption by components other than alumina. Therefore, the lower diffraction intensities mean stronger X-ray absorption by 

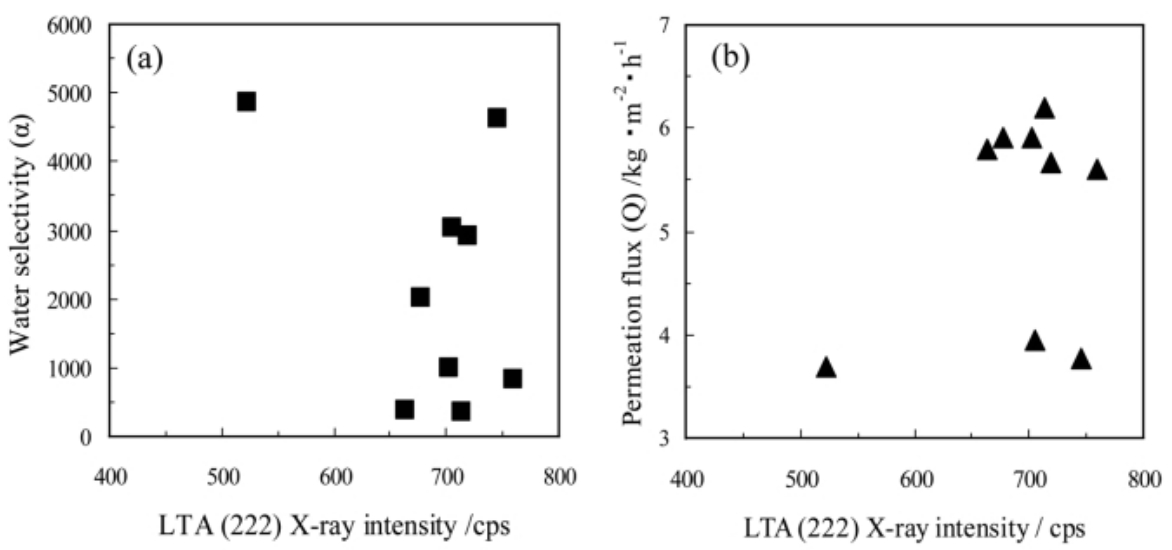

Fig. 2 Relationships among the LTA (222) diffraction intensity and the water selectivity (a), and the permeation flux (b) of the LTA membrane.
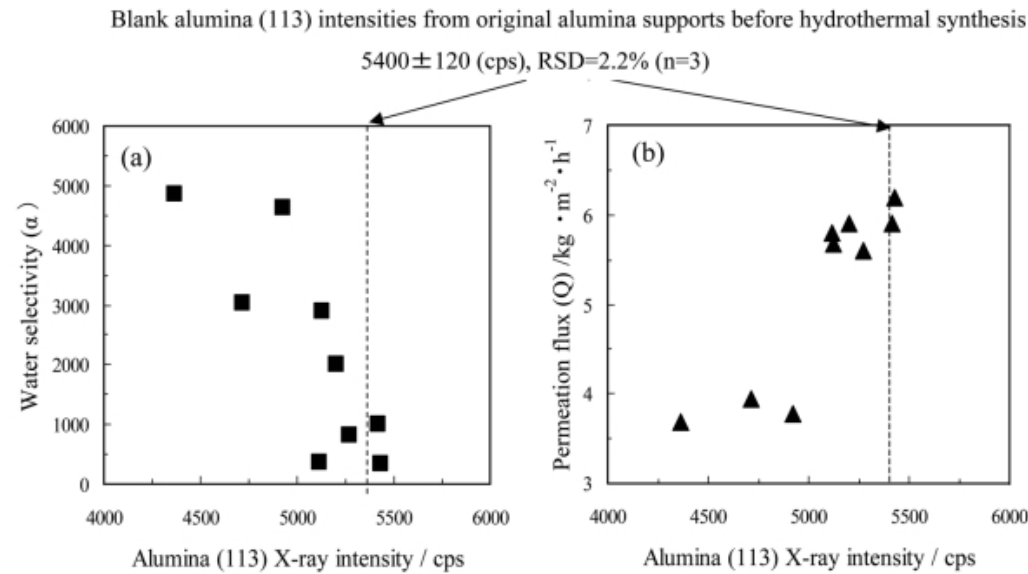

Fig. 3 Relationships among the alumina (113) diffraction intensity and the water selectivity (a), and the permeation flux (b) of the LTA membrane.

the LTA membrane overall. As shown in Fig. 1(b), although the thicknesses of the surface layer of the LTA membranes used in this study were approximately $2-3 \mu \mathrm{m}$, and changed little among the different membranes, those of materials embedded in the alumina porous support were very thick. This time, the real thicknesses of materials embedded in the alumina porous support were not determined, because the alumina support layer is very hard, so that FIB operation is time-consuming. Also, in our previous work, ${ }^{3,4}$ it has been suggested that materials embedded in an alumina porous support consist of both the LTA crystal and amorphous-like substances. The overall absorption coefficient of materials embedded in an alumina porous support should be almost the same as that of the surface LTA crystal. Therefore, a major factor causing the difference in the X-ray absorption is a difference in the thickness or quantity of materials embedded in the alumina porous support, rather than those of the surface LTA crystal. However, a further excavation of the alumina support layer by FIB is also needed to confirm the present X-ray absorption.

In conclusion, a relationship between the X-ray absorption and the PV dehydration performance of the LTA membrane was considered for the first time. By using the relationship, the $\mathrm{EtOH} / \mathrm{H}_{2} \mathrm{O}$ PV performance (water selectivity and permeation flux) of the LTA membrane can be conveniently estimated without real PV experiments. Thus, the present XRD study can provide a novel discussion on water permeation in the LTA membrane.

\section{References}

1. T. C. Bowen, R. D. Noble, and J. L. Falconer, J. Membr. Sci., 2004, 245, 1, and references therein.

2. A. Jentys and J. A. Lercher, "8. Techniques of zeolite characterization", in "Introduction to Zeolite Science and Practice", ed. H. van Bekkum, E. M. Flanigen, P. A. Jacobs, and J. C. Jansen, 2nd ed., 2001, Elsevier Science, Amsterdam, 345 - 386, and references therein.

3. T. Kyotani, K. Sato, T. Mizuno, S. Kakui, M. Aizawa, J. Saito, S. Ikeda, S. Ichikawa, and T. Nakane, Anal. Sci., $\mathbf{2 0 0 5}, 21,321$.

4. T. Kyotani, S. Kakui, and J. Saito, 85th Annual Meeting of the Chemical Society of Japan, Abstract, 2005, Yokohama 2A5-47.

5. S. Ichikawa, S. Tanaka, T. Nakane, M. Aizawa, K. Sato, T. Mizuno, and H. Chida, 18th Annual Meeting of the Japan Association of Zeolites, Abstract, 2002, 56 - 57.

6. T. Mizuno and K. Sato, Japan Patent, 2004, JP2004082008A 